\section{Regulatory role of the JAK STAT kinase signalling system on the IL-23/L-17 cytokine axis in psoriatic arthritis}

We read with great interest the article by Gao et al. ${ }^{1}$ They have reported the regulatory role of the JAK/STAT kinase system on the inflammatory/proliferative cascades for pannus formation in psoriatic arthritis (PsA) such as on fibroblast like synovial cells (FLS) biology and on secretion of inflammatory cytokines (interleukin (IL) 6, IL-8, monocyte chemoattractant protein (MCP)-1) by these FLS. Tofacitinib targets JAK1 and JAK2 with IC50 values in the same order of magnitude as that of JAK3. ${ }^{2}$ They have also provided the mechanisms of actions of tofacitinib by demonstrating that tofacitinib significantly decreased pSTAT3, pSTAT1, NFkBp65 in PsAFLS and inhibits the cellular and molecular events of pannus formation. However, Gao et al did not address a critical issue whether JAK/STAT signalling system regulates the IL-23/IL-17 cytokine axis in PsA. Here we are sharing an alternative mechanism for the role of JAK/STAT kinase system in the pathogenesis of PsA.

Aberrant activation of IL-23/IL-17 cytokine axis is a dominant pathology in PsA. ${ }^{3}{ }^{4}$ JAK2 is recruited to IL-23 receptor, so it is expected that JAK/STAT-mediated signalling system is important in PsA. We hypothesised- (i) JAK/STAT signalling system regulates the Th17 cells in PsA and (ii) that tofacitinib which inhibits Jak-2 likely targets the Th17 cells by inhibiting the IL-23-induced JAK/STAT signalling system.

\section{METHODS}

Mononuclear cells of peripheral blood (PBMC) and synovial fluid (SFMC) from patients with PsA $(n=15)$ and PBMC from age/sex matched normal individuals $(n=15)$ were collected. All patients had an active disease and were not on disease modifying anti-rheumatic drugs (DMARDS) or biologics. Recombinant IL-23 (rIL-23) (40 ng/mL) induced activated IL-17+ T cells were generated and evaluated as per our earlier reports. ${ }^{3}$ Cells were cultured with and without tofacitinib $(50 \mathrm{nM})$. Western
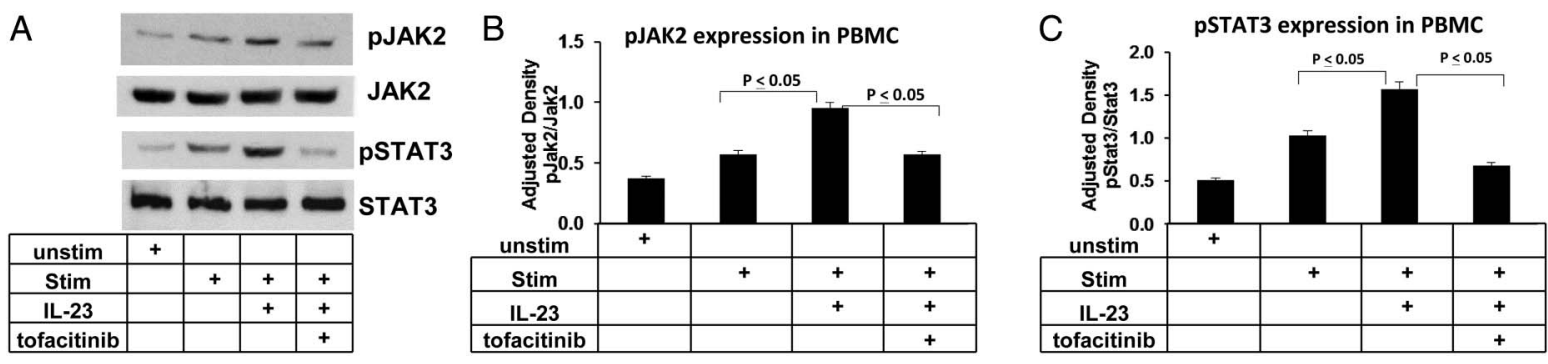

Figure 1 Interleukin (IL) 23 induces phosphorylation of JAK2 and STAT3 in mononuclear cells of peripheral blood (PBMC) of psoriatic arthritis (PsA) and that can be inhibited by tofacitinib. Results of the (A-C) demonstrate that IL-23 induced phosphorylation of JAK2 and STAT3 ( $<<0.05$, $t$-test). Tofacitinib significantly inhibited activation of these signalling proteins ( $p<0.05$, $t$-test). Experiments were done in triplicate and the results were expressed in mean \pm SD of the adjusted density.
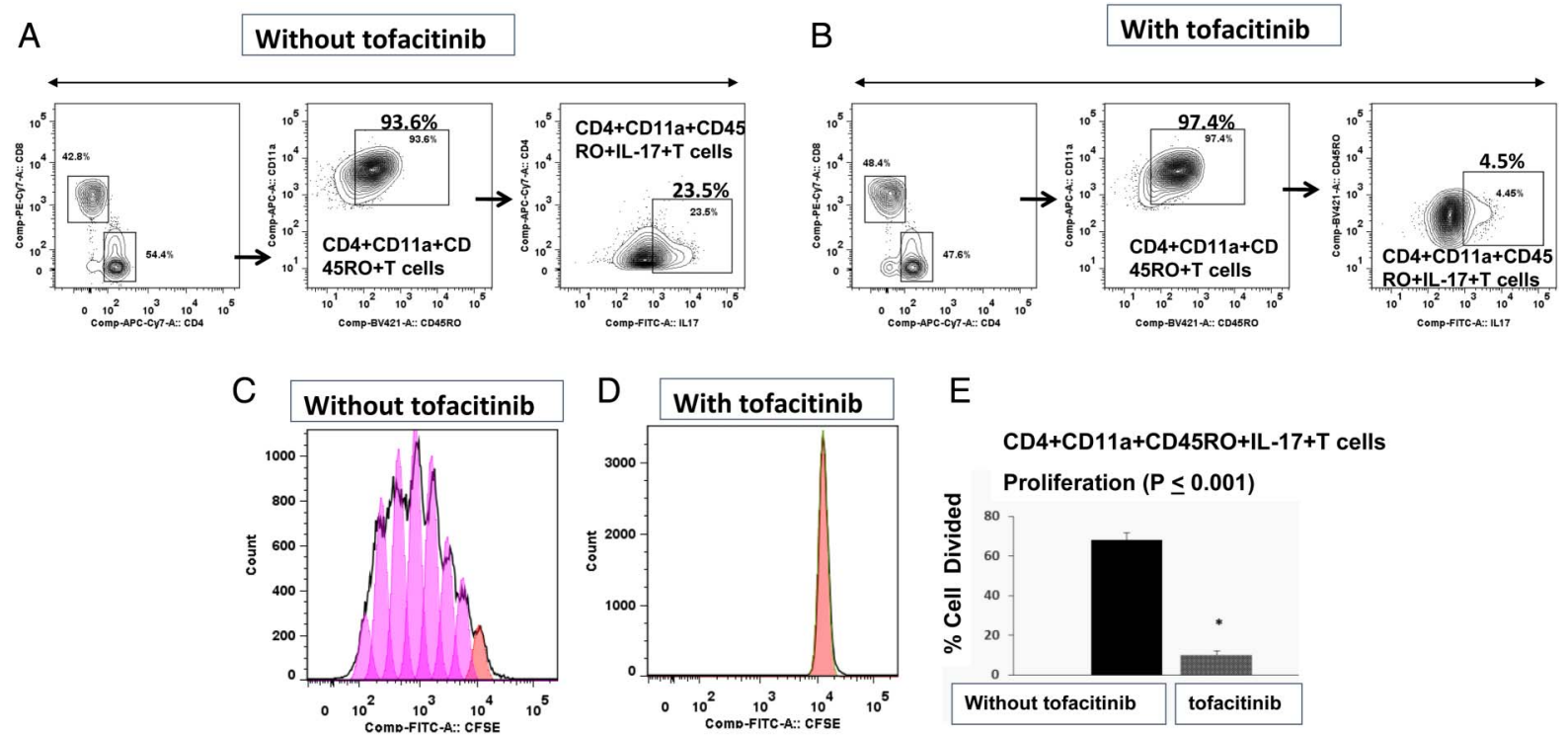

Figure 2 (A, B) Hi-D FACS studies of the mononuclear cells of peripheral blood from patients with psoriatic arthritis demonstrated that- (i) rlL-23 induced marked upregulation of IL-17 in the CD4+ memory T cells (CD11a+CD45RO+) (A) and that (ii) rIL-23 induced IL-17 expression could be markedly inhibited by tofacitinib $(p<0.001$, t-test) (B). Representative FACS plots are shown here and results are described in the text. (C-E) To determine the effect of the JAK-STAT kinase system on the proliferation of CD4+CD11a+CD45RO+IL-17+ T cells we performed the CFSE assay specifically on this T cell subpopulation. The bar diagram demonstrates that rlL-23 induced marked proliferation of the CD4+CD11a+CD45RO+IL-17 $+T$ cells and tofacitinib significantly inhibited proliferation of these pathological cells $(p<0.001, t$-test) (E). Representative FACS plots demonstrate less number of generations and less numbers of CD4+CD11a+CD45R0+IL-17+ T cells on day 5 in PBMC cultured with tofacitinib (D) compared with cells cultured without tofacitinib (C). CFSE, carboxyfluorescein succinimidyl ester; IL, interleukin; rlL, recombinant IL. 
blot studies were performed to indentify Jak2/p-Jak2 and stat $3 /$ p-stat 3 in the sorted activated CD3 + T cells. Hi-D fluorescenceactivated cell sorting (FACS) studies were performed to identify the activated memory CD4+CD11a+CD45RO+IL-17+ T cells and CD8 +CD11a+CD45RO+IL-17+ T cells in SFMC/ PBMC of PsA and PBMC of normal individuals.

\section{RESULTS}

In both PsA and controls sorted activated CD3 $+\mathrm{T}$ cells in the presence of IL-23 demonstrated activation of Jak2 and STAT3. Further, we noticed tofacitinib markedly inhibited phosphorylation of Jak2 and STAT-3, the signalling proteins induced by IL-23 (figure $1 \mathrm{~A}-\mathrm{C}$ ).

Hi-D FACS analyses of the activated CD3 + T cells in patients with PsA demonstrated that IL-23 induced marked upregulation of IL-17 in the memory $\mathrm{T}$ cells (CD11a+CD45RO+) (figure 2A). We noticed that SFMC and PBMC treated with rIL-23 in patients with PsA had $30 \pm 4.5 \%$ and $18 \pm 3.8 \%$ activated memory CD4+IL-17+ T cells, respectively, compared with $5 \pm 0.7 \%$ in healthy persons $(\mathrm{p}<0.001 \%)$. Further, we noticed that CD4+CD11a+CD45RO+IL-17+ T cells were $5 \pm 2 \%(\mathrm{p}<0.001 \%)$ in cells treated with Tofacitinib (figure $2 \mathrm{~B}$ ). Tofacitinib also significantly inhibited proliferation of these CD4 $+\mathrm{CD} 11 \mathrm{a}+\mathrm{CD} 45 \mathrm{RO}+\mathrm{IL}-17+\mathrm{T}$ cells $(\mathrm{p}<0.001 \%)$ (figure 2E).

\section{CONCLUSION}

Th17 cells play a critical role in the pathogenesis of PsA. ${ }^{3} 4$ Here, we observed that the generation of these pathological $\mathrm{CD} 4+\mathrm{CD} 11 \mathrm{a}+\mathrm{CD} 45 \mathrm{RO}+\mathrm{IL}-17+\mathrm{T}$ cells and their proliferation are regulated by the JAK-STAT signalling system. A plausible mechanism of action of tofacitinib likely to be inhibition of the IL-23/IL-17 cytokine axis by inhibiting the IL-23-induced JAK-STAT signalling system.

\section{Smriti K Raychaudhuri, ${ }^{1}$ Christine Abria, ${ }^{1}$ Siba P Raychaudhuri ${ }^{1,2}$}

${ }^{1}$ VA Medical Center Sacramento, Davis, California, USA
${ }^{2}$ Division of Rheumatology, Allergy \& Clinical immunology, University of California School of Medicine, Davis, California, USA

Correspondence to Dr Siba P Raychaudhuri, Division of Rheumatology, Allergy \& Clinical Immunology, University of California School of Medicine, Davis, Chief of Rheumatology, VA Medical Center Sacramento, 10535 Hospital Way, Bldg\#650, Research Service, Mather, CA 95655, USA; sraychaudhuri@ucdavis.edu

Contributors SKR: Reviewed and analysed data, helped to prepare the manuscript. CA: Performed experiments, analysed data and helped to prepare the manuscript. SPR: Designed the study, reviewed and analysed data, helped to prepare the manuscript.

Funding This project was supported by the VA Medical Center Sacramento.

Competing interests None declared.

Ethics approval IRB-VA Sacramento Medical Center.

Provenance and peer review Not commissioned; internally peer reviewed.

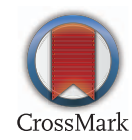

To cite Raychaudhuri SK, Abria C, Raychaudhuri SP. Ann Rheum Dis 2017;76:e36.

Received 29 December 2016

Accepted 3 January 2017

Published Online First 8 February 2017

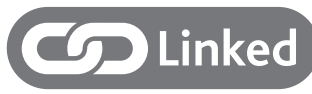

http://dx.doi.org/10.1136/annrheumdis-2017-211081

Ann Rheum Dis 2017;76:e36. doi:10.1136/annrheumdis-2016-211046

REFERENCES

1 Gao W, McGarry T, Orr C, et al. Tofacitinib regulates synovial inflammation in psoriatic arthritis, inhibiting STAT activation and induction of negative feedback inhibitors. Ann Rheum Dis 2016;75:311-15.

2 Meyer D M, Jesson MI, Li X, et al. Anti-inflammatory activity and neutrophil reductions mediated by the JAK1/JAK3 inhibitor, CP-690,550, in rat adjuvant-induced arthritis. J Infl amm (Lond) 2010;7:41.

3 Raychaudhuri SP, Raychaudhuri SK, Genovese MC. IL-17 receptor and its functional significance in psoriatic arthritis. Mol Cell Biochem 2012;359:419-29.

4 Raychaudhuri SP. Role of IL-17 in psoriasis and psoriatic arthritis. Clin Rev Allergy Immunol 2013;44:183-93. 